\title{
Genome Wide Association Analysis of the 16th QTL- MAS Workshopdataset using the Random Forest machine learning approach
}

\author{
Giulietta Minozzi ${ }^{1,3 *}$, Andrea Pedretti ${ }^{1}$, Stefano Biffani ${ }^{2}$, Ezequiel Luis Nicolazzi ${ }^{1}$, Alessandra Stella ${ }^{2}$ \\ From 16th QTL-MAS Workshop \\ Alghero, Italy. 24-25 May 2012
}

\begin{abstract}
Background: Genome wide association studies are now widely used in the livestock sector to estimate the association among single nucleotide polymorphisms (SNPs) distributed across the whole genome and one or more trait. As computational power increases, the use of machine learning techniques to analyze large genome widedatasets becomes possible.

Methods: The objective of this study was to identify SNPs associatedwith the three traits simulated in the 16th MAS-QTL workshop dataset using the Random Forest (RF) approach. The approach was applied to single and multiple trait estimated breeding values, and on yield deviations and to compare them with the results of the GRAMMAR-CG method.
\end{abstract}

Results: The two QTL mapping methods used, GRAMMAR-CG and RF, were successful in identifying the main QTLs for trait 1 on chromosomes 1 and 4, for trait 2 on chromosomes 1, 4 and 5 and for trait 3 on chromosomes 1,2 and 3 .

Conclusions: The results of the RF approach were confirmed by the GRAMMAR-CG method and validated by the effective QTL position, even if their approach to unravel cryptic genetic structure is different. Furthermore, both methods showed complementary findings. However, when the variance explained by the QTL is low, they both failed to detect significant associations.

\section{Background}

Genome wide association studies (GWAs) are now widely used in the livestock sector to estimate the association among multiple single nucleotide polymorphisms (SNPs) distributed across the whole genome and one or more trait. GWAs are typically carried out on a singlepoint by performing a marginal chi-square test or regression. However, these methods do not take into account linkage disequilibrium between markers and the genetic structure of the population that may have a large impact on structured populations (e.g. cattle populations). Approaches for genome wide pedigree-based quantitative trait loci (QTL) analysis have been developed (e.g. GRAMMAR-CG), which are based on mixed

\footnotetext{
* Correspondence: Giulietta.minozzi@tecnoparco.org

'Parco Tecnologico Padano Srl, Via Einstein 26900 Lodi, Italy

Full list of author information is available at the end of the article
}

model and regression, where the genomic kinship matrix estimated through genomic marker data can be used to correct for familiar correlation and cryptic relatedness [1].

As computational power increases, the use of more advanced machine learning techniques to analyze large genome wide datasets becomes possible [2], these techniques include Support Vector Machines [3], Bayesian Networks [4] and Random Forest [5].

The Random Forests (RF) algorithm [6] is a machinelearning method that has been widely applied to classification and regression problems, and is particularly well suited to circumstances in which the number of potential explanatory variables exceeds the number of observations, as is the case for GWAs. The RF algorithm produces a collection of trees (forest), each grown on a different bootstrap sample of observations, and at each 
split (node) of a tree, a different random subset of predictors (SNP) is evaluated to identify the best split. The final scores are then calculated by aggregating predictions resulting from all the trees grown in the forest.

RF embraces a combination of characteristics that makes it appropriate for genetic applications: it is well suited for very large datasets; it is non-parametric, thus does not require a causal model to be specified, it is highly parallelizable and considers interactions between predictors.

The objective of this study was to identify SNPs associated to the three traits simulated in the 16th MASQTL workshop dataset using the Random Forest approach and to compare them with the results obtained by the Grammar-CG method. SNPs identified by both methods were verified with the actual QTL positions.

\section{Methods}

\section{Dataset}

The dataset used was provided by the organisers of the 16th QTLMAS workshop and consisted of 4080 individuals (G0 to G4). The simulated genome was $499.750 \mathrm{Mb}$ consisting of 5 chromosomes carrying 2,000 equally distributed SNPs. The GWA analysis was conducted on 3000 samples, all females belonging to generations G1 to G3, for which phenotypic information for three traits (yield deviations) was provided. The analysis was performed on: yield deviations (YD1, YD2 and YD3), the estimated breeding values (EBV) obtained from a single trait model (tr1_ST, tr2_ST, tr3_ST) and the EBVs obtained from a multiple trait model (tr1_MT, tr2_MT, tr3_MT).

\section{Analysis}

\section{Variance components and EBV estimation}

Variance components and EBVs were obtained separately, using REMLF90 and BLUPF90 programs, respectively [7]. The model used to estimate variance components and EBVs was:

$$
\gamma_{k, i, j}=\mu_{k}+G_{k, i}+\text { Animal }_{k, j}+e_{k, i, j}
$$

where $\mu$ is a general mean for the $k^{\text {th }}$ trait, GEN is a fixed effect for i generations ( $i=1$ to 3 ), Animal is a random animal effect with distribution $\sim \mathrm{N}\left(0, \sigma^{2}{ }_{\mathrm{a}}\right)$, where $\sigma^{2}{ }_{a}$ is the additive genetic variance, and $e$ is the random residual with distribution $\sim \mathrm{N}\left(0, \sigma^{2}{ }_{\mathrm{e}}\right)$, where $\sigma^{2}{ }_{\mathrm{e}}$ is the residual variance. Covariance between traits was considered only in multiple-trait analysis.

\section{Random Forest}

Feature selection (SNPs) analysis was performed with the randomForest package in $\mathrm{R}$ [8] using 3000 individuals and the 9042 SNPs that passed quality control checks out of the total 10000 SNP. The minimum size of the terminal nodes was set to 5 . The number of trees grown was set to 1000 . The subset of samples evaluated at each tree was $70 \%$ of the total number of samples $(n=2100)$. The number of variables evaluated at each node was set to the square root of the number of predictors $(\mathrm{p}=94)$. All SNPs were ordered by Mean decrease Gini index [6] and the most strongly associated SNPs are at the top of the lists shown in Table 2, 3 and 4.

\section{Grammar-CG}

Genome-wide association analysis was performed with the GenABEL package in R using a three step GRAMMAR-CG (Genome wide Association using Mixed Model and Regression - Genomic Control) approach [1,9].

\section{Results and discussion}

Variance components and EBV estimation

Mean and standard deviations of the nine phenotypes used are shown in Table 1 . The heritability estimates resulting from the single trait model were $0.38,0.38$ and 0.50 for trait 1,2 and 3, respectively. Large genetic correlations between traits 1 and 2 were observed (0.83), whereas lower genetic correlation was observed for trait 2 and 3 (0.12). Negative correlation was observed between traits 1 and $3(-0.44)$.

\section{Association mapping}

The two QTL mapping methods used, GRAMMAR-CG and RF, were successful in identified the largest QTLs for trait 1 on chromosomes 4 and 1 in position $24 \mathrm{Mb}$ and $84 \mathrm{Mb}$, for trait 2 on chromosomes 4,5 and 1 in position $24 \mathrm{Mb}, 68 \mathrm{Mb}$ and $14 \mathrm{Mb}$ and for trait 3 on chromosomes 1, 2 and 3 at $84 \mathrm{Mb}, 79 \mathrm{Mb}$ and $36 \mathrm{Mb}$ respectively. Positions and names of the significant SNPs are shown in Tables 2, 3 and 4.

Both methods showed good precision in the identification of the QTL in comparison with the "real" QTL

\section{Table 1 Statistics of the nine phenotypes used in the} GWAs.

\begin{tabular}{cccc}
\hline Trait & $\mathbf{n}$ & Mean & Sd \\
\hline YD1 & 3000 & 0 & 176,519 \\
YD2 & 3000 & 0 & 9,512 \\
YD3 & 3000 & 0 & 0,024 \\
tr1_MT & 3000 & $-0,238$ & 81,495 \\
tr2_MT & 3000 & 0,031 & 4,264 \\
tr3_MT & 3000 & 0 & 0,015 \\
tr1_ST & 3000 & $-0,555$ & 79,057 \\
tr2_ST & 3000 & 0,0041 & 4,254 \\
tr3_ST & 3000 & 0 & 0,014 \\
\hline
\end{tabular}

Yield deviations for the three traits (YD1, YD2 and YD3), estimated breeding values (EBV) obtained from a single trait model (tr1_ST, tr2_ST, tr3_ST) and from a multiple trait model (tr1_MT, tr2_MT, tr3_MT). 
Table 2 Top SNPs identified by the Random Forest and GRAMMAR-CG Approach for Trait 1

\begin{tabular}{|c|c|c|c|c|c|c|}
\hline \multicolumn{7}{|c|}{ Trait 1} \\
\hline \multicolumn{3}{|c|}{ Random Forest method } & \multicolumn{3}{|c|}{ Grammar_CG } & \\
\hline \multicolumn{3}{|c|}{ Multiple Trait EBV } & \multicolumn{3}{|c|}{ Multiple Trait EBV } & \\
\hline SNPname & $\mathrm{CHR}$ & pos Kb & name & CHR & pos $\mathrm{Kb}$ & $\mathrm{p}$-value \\
\hline SNP6499 & 4 & 24.900 & SNP6499 & 4 & 24.900 & $3,6 \mathrm{E}-17$ \\
\hline SNP4688 & 3 & 34.350 & SNP1682 & 1 & 84.050 & $1,2 \mathrm{E}-07$ \\
\hline SNP4674 & 3 & 33.650 & SNP1683 & 1 & 84.100 & $4,4 \mathrm{E}-06$ \\
\hline SNP4197 & 3 & 9.800 & SNP6498 & 4 & 24.850 & $7,2 \mathrm{E}-06$ \\
\hline SNP7145 & 4 & 57.200 & SNP3585 & 2 & 79.200 & $1,1 \mathrm{E}-05$ \\
\hline SNP1012 & 1 & 50.550 & SNP6501 & 4 & 25.000 & $1,1 \mathrm{E}-05$ \\
\hline SNP1614 & 1 & 80.650 & SNP6469 & 4 & 23.400 & $8,4 \mathrm{E}-05$ \\
\hline SNP6534 & 4 & 26.650 & SNP9362 & 5 & 68.050 & $9,5 \mathrm{E}-05$ \\
\hline \multicolumn{3}{|c|}{ Single Trait EBV } & \multicolumn{3}{|c|}{ Single trait EBV } & \\
\hline SNP6499 & 4 & 24.900 & SNP6499 & 4 & 24.900 & $9,8 \mathrm{E}-16$ \\
\hline SNP4688 & 3 & 34.350 & SNP1682 & 1 & 84.050 & $9,8 \mathrm{E}-06$ \\
\hline SNP4674 & 3 & 33.650 & SNP6501 & 4 & 25.000 & $1,4 \mathrm{E}-05$ \\
\hline SNP4197 & 3 & 9.800 & SNP6498 & 4 & 24.850 & $3,0 \mathrm{E}-05$ \\
\hline SNP1012 & 1 & 50.550 & SNP1683 & 1 & 84.100 & $5,0 \mathrm{E}-05$ \\
\hline SNP1614 & 1 & 80.650 & SNP293 & 1 & 14.600 & $5,6 \mathrm{E}-05$ \\
\hline \multicolumn{3}{|c|}{ Yield Deviation } & \multicolumn{3}{|c|}{ Yield Deviation } & \\
\hline SNP6499 & 4 & 24.900 & SNP6499 & 4 & 24.900 & $1,9 \mathrm{E}-19$ \\
\hline SNP1683 & 1 & 84.100 & SNP1682 & 1 & 84.050 & $4,2 \mathrm{E}-09$ \\
\hline SNP6507 & 4 & 25.300 & SNP1683 & 1 & 84.100 & $2,8 \mathrm{E}-08$ \\
\hline SNP1614 & 1 & 80.650 & SNP6498 & 4 & 24.850 & $2,7 \mathrm{E}-07$ \\
\hline SNP6506 & 4 & 25.250 & SNP6501 & 4 & 25.000 & $6,9 \mathrm{E}-07$ \\
\hline SNP4674 & 3 & 33.650 & SNP6506 & 4 & 25.250 & $3,3 \mathrm{E}-06$ \\
\hline SNP1682 & 1 & 84.050 & SNP293 & 1 & 14.600 & $9,2 \mathrm{E}-06$ \\
\hline SNP9374 & 5 & 68.650 & SNP6507 & 4 & 25.300 & $2,7 \mathrm{E}-05$ \\
\hline SNP1012 & 1 & 50.550 & SNP1699 & 1 & 84.900 & $5,1 \mathrm{E}-05$ \\
\hline SNP1685 & 1 & 84.200 & SNP1161 & 1 & 58.000 & $7,6 \mathrm{E}-05$ \\
\hline
\end{tabular}

Table 3 Top SNPs identified by the Random Forest and GRAMMAR-CG Approach for trait 2

\begin{tabular}{|c|c|c|c|c|c|c|}
\hline \multicolumn{7}{|c|}{ Trait 2} \\
\hline \multicolumn{3}{|c|}{ Random Forest method } & \multicolumn{3}{|c|}{ Grammar_CG } & \\
\hline \multicolumn{3}{|c|}{ Multiple Trait EBV } & \multicolumn{3}{|c|}{ Multiple Trait EBV } & \\
\hline SNP name & CHR & pos Kb & SNP name & CHR & Pos Kb & p-value \\
\hline SNP6499 & 4 & 24.900 & SNP6499 & 4 & 24.900 & $1,93 \mathrm{E}-18$ \\
\hline SNP7151 & 4 & 57.500 & SNP293 & 1 & 14.600 & $3,51 \mathrm{E}-10$ \\
\hline SNP298 & 1 & 14.850 & SNP4044 & 3 & 2.150 & $6,38 \mathrm{E}-10$ \\
\hline SNP2171 & 2 & 8.500 & SNP298 & 1 & 14.850 & $4,07 \mathrm{E}-07$ \\
\hline SNP293 & 1 & 14.600 & SNP6501 & 4 & 25.000 & $1,79 \mathrm{E}-06$ \\
\hline SNP9528 & 5 & 76.350 & SNP6498 & 4 & 24.850 & $1,74 \mathrm{E}-05$ \\
\hline SNP296 & 1 & 14.750 & SNP296 & 1 & 14.750 & $8,60 \mathrm{E}-05$ \\
\hline \multicolumn{3}{|c|}{ Single Trait EBV } & \multicolumn{3}{|c|}{ Single Trait EBV } & \\
\hline SNP6499 & 4 & 24.900 & SNP6499 & 4 & 24.900 & $1,93 \mathrm{E}-19$ \\
\hline SNP7151 & 4 & 57.500 & SNP293 & 1 & 14.600 & $1,74 \mathrm{E}-09$ \\
\hline SNP2171 & 2 & 8.500 & SNP4044 & 3 & 2.150 & $1,24 \mathrm{E}-08$ \\
\hline SNP298 & 1 & 14.850 & SNP298 & 1 & 14.850 & $1,12 \mathrm{E}-06$ \\
\hline SNP293 & 1 & 14.600 & SNP6501 & 4 & 25.000 & $1,17 \mathrm{E}-06$ \\
\hline SNP9528 & 5 & 76.350 & SNP6498 & 4 & 24.850 & 8,61E-06 \\
\hline
\end{tabular}


Table 3 Top SNPs identified by the Random Forest and GRAMMAR-CG Approach for trait 2 (Continued)

\begin{tabular}{|c|c|c|c|c|c|c|}
\hline \multicolumn{3}{|c|}{ Yield Deviation } & \multicolumn{3}{|c|}{ Yield Deviation } & \multirow[b]{2}{*}{$2,90 \mathrm{E}-24$} \\
\hline SNP6499 & 4 & 24.900 & SNP6499 & 4 & 24.900 & \\
\hline SNP293 & 1 & 14.600 & SNP293 & 1 & 14.600 & $1,21 \mathrm{E}-11$ \\
\hline SNP298 & 1 & 14.850 & SNP6501 & 4 & 25.000 & $2,34 \mathrm{E}-08$ \\
\hline SNP296 & 1 & 14.750 & SNP298 & 1 & 14.850 & $4,93 \mathrm{E}-08$ \\
\hline SNP6507 & 4 & 25.300 & SNP6498 & 4 & 24.850 & $7,76 \mathrm{E}-08$ \\
\hline SNP6506 & 4 & 25.250 & SNP4044 & 3 & 2.150 & $3,19 \mathrm{E}-07$ \\
\hline SNP6425 & 4 & 21.200 & SNP296 & 1 & 14.750 & $1,58 \mathrm{E}-06$ \\
\hline SNP9374 & 5 & 68.650 & SNP6506 & 4 & 25.250 & $2,80 \mathrm{E}-06$ \\
\hline SNP295 & 1 & 14.700 & SNP295 & 1 & 14.700 & $3,81 \mathrm{E}-06$ \\
\hline \multirow[t]{5}{*}{ SNP7151 } & 4 & 57.500 & SNP6503 & 4 & 25.100 & $1,66 \mathrm{E}-05$ \\
\hline & & & SNP6507 & 4 & 25.300 & $2,37 \mathrm{E}-05$ \\
\hline & & & SNP6504 & 4 & 25.150 & $5,45 \mathrm{E}-05$ \\
\hline & & & SNP6502 & 4 & 25.050 & $8,14 \mathrm{E}-05$ \\
\hline & & & SNP9362 & 5 & 68.050 & $8,14 \mathrm{E}-05$ \\
\hline
\end{tabular}

Table 4 Top SNPs identified by the Random Forest and GRAMMAR-CG Approach for trait 3

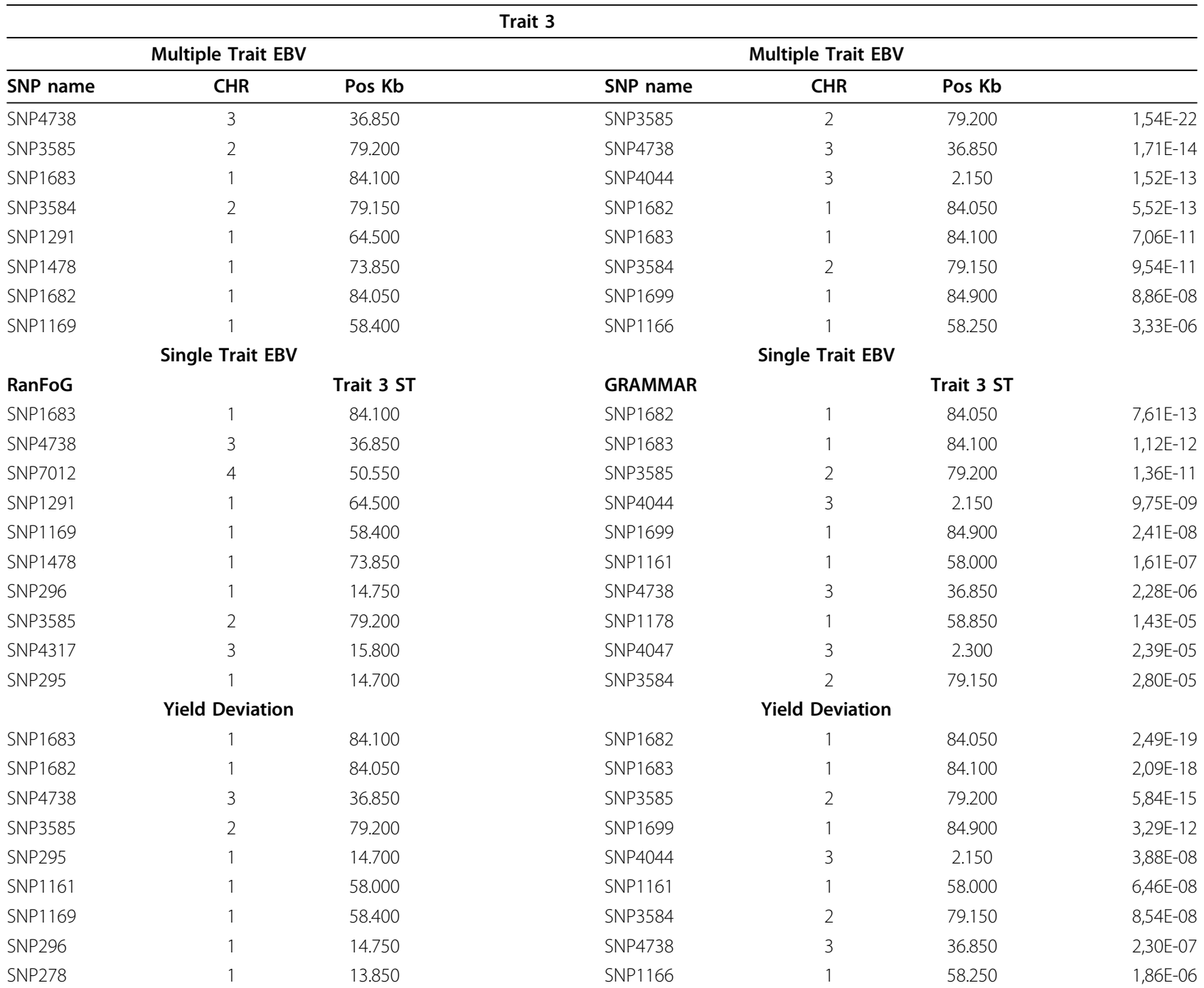


Table 4 Top SNPs identified by the Random Forest and GRAMMAR-CG Approach for trait 3 (Continued)

\begin{tabular}{|c|c|c|c|c|c|c|}
\hline \multirow[t]{6}{*}{ SNP1166 } & 1 & 58.250 & SNP1697 & 1 & 84.800 & $1,92 \mathrm{E}-06$ \\
\hline & & & SNP1178 & 1 & 58.850 & $3,32 \mathrm{E}-06$ \\
\hline & & & SNP1168 & 1 & 58.350 & 7,79E-06 \\
\hline & & & SNP1685 & 1 & 84.200 & $1,42 \mathrm{E}-05$ \\
\hline & & & SNP3595 & 2 & 79.700 & $2,08 \mathrm{E}-05$ \\
\hline & & & SNP4047 & 3 & 2.300 & $3,11 \mathrm{E}-05$ \\
\hline
\end{tabular}

position provided by [10]. Interestingly the exact markers flankingthe QTL were identified for all traits.

Differences however were observed depending on i) the phenotype analysed, YD, single trait EBV and Multiple trait EBV and on ii) the method used RF or GRAMMAR-CG.

With regard to Trait 1, the GRAMMAR-CG method identified 8 significant associations for multiple trait EBV, 6 for single trait EBVs and 10 for YD, only 4 of which are common between the three phenotypes. The RF approach identified the same number of markers per phenotype, but only 2 markers were in common between the methods of analysis and phenotype. The two markers identified by both approaches were the QTL which explained the largest variance, however, the other markers are all true associations and indicate that using different types of phenotypes for the same trait and different analysis methods may overlap, but may also show some differences in QTLs and positions.

Traits 2 and 3 share the same pattern as observed for trait 1. Several QTL were identified in common between phenotypes and methods but just a few were in common between analysis methods: 2 markers for trait 2 and 3 markers for trait 3 . When the YD phenotype was used, a larger number of significant SNPs were detected. This may be due to the larger variability of the YD compared to the more regressed EBV phenotypes (Table 1).

Interestingly both methods failed to identify the QTLs on chromosomes 4 and 5 for Trait 3. The variance explained by the markers is low, suggesting that both methods are not able to detect QTLs which explain a small amount of variance. The RF approach, however, detect the QTL on chromosomes 5 and 3 for Trait 1 .

Overall the results of the RF were confirmed by the results of the GRAMMAR-CG method and were validated by the effective positions given the QTL. Interestingly, even though the RF approach does not directly use family structure information through a relationship matrix (genomic or additive), as is the case in the GRAMMAR-CG approach, correct identification of QTL positions is achieved.

\section{Conclusions}

In this study we proposed the use of recursive partitioning approaches such as Random Forest, as an alternative to traditional regression methods to detect the genetic loci. The results of the RF approach were consistent with those of the GRAMMAR-CG method and validated by the effective positions given for the QTL. However, when the variance explained by the QTL was low, both failed to detect a significant association.

\section{Competing interests}

The authors declare that they have no competing interests.

\section{Authors' contributions}

AP, GM, ELN, and SB participated in the design and performed the statistical analysis. GM and AS conceived the study, participated in its design and coordination. GM drafted the manuscript. All authors read and approved the final manuscript.

\section{Acknowledgements}

This work was funded by the MASTFIELD project n.1745 (Applicazione di sistemi molecolari innovativi per ilcontrollo in campo delle mastiti bovine) of the Lombardy Region(Agricultural regional research programme2010-2012) and by the PON EPISUD project n PON01_ 01841 project funded by MIUR (Ministero dell'Istruzione, dell'Università e della Ricerca).

\section{Declarations}

This work was funded by the MASTFIELD project n.1745 (Applicazione di sistemi molecolari innovativi per ilcontrollo in campo delle mastiti bovine) of the Lombardy Region (Agricultural regional research programme2010-2012) and by the PON EPISUD project n PON01_ 01841 project funded by MIUR (Ministero dell'Istruzione, dell'Università e della Ricerca).

This article has been published as part of BMC Proceedings Volume 8 Supplement 5, 2014: Proceedings of the 16th European Workshop on QTL Mapping and Marker Assisted Selection (QTL-MAS). The full contents of the supplement are available online at http://www.biomedcentral.com/bmcproc/ supplements/8/S5

\section{Authors' details}

${ }^{1}$ Parco Tecnologico Padano Srl, Via Einstein 26900 Lodi, Italy. ${ }^{2}$ IBBA-CNR, Via Einstein 26900, 26900 Lodi, Italy. ${ }^{3}$ Department of Veterinary Science and Public Health, University of Milan, Via Celoria 10, 20133 Milan, Italy.

Published: 7 October 2014

\section{References}

1. Amin N, van Duijn CM, Aulchenko YS: A genomic background based method for association analysis in related individuals. PLOS ONE 2007, 2: e1274.

2. Goldstein BA, Polley EC, Briggs FB: Random forests for genetic association studies. Stat Appl Genet Mol Biol 2011, 10(1):32.

3. Yoon Y, Song J, Hong S, Kim J: Analysis of multiple single nucleotide polymorphisms of candidate genes related to coronary heart disease susceptibility by using support vector machines. Clin Chem Lab Med 2003, 41:529-534.

4. Han B, Chen XW, Talebizadeh Z, Xu H: Genetic studies of complex human diseases: characterizing SNP-disease associations using Bayesian networks. BMC Syst Biol 2012, 6. 
5. Mokry FB, Higa RH, de Alv arenga Mudadu M, Oliveira de Lima A, Meirelles SL, Barbosa da Silva MV, Cardoso FF, Morgado de Oliveira M Urbinati I, Méo Niciura SC, Tullio RR, Mello de Alencar M, Correia de Almeida Regitano L: Genome-wide association study for backfat thickness in Canchim beef cattle using Random Forest approach. BMC Genet 2013, 14:47, Jun 5.

6. Breiman L: 2001 Random Forests. Machine Learning 2001, 45:5-32

7. Misztal I, Tsuruta S, Strabel T, Auvray B, Druet T, Lee DH: BLUPF90 and related programs (BGF90). Proc. 7th WCGALP Montpellier 2002, France; Communication No. 28-07.

8. [http://cran.r-project.org/web/packages/randomForest/index.html]

9. Aulchenko YS, Ripke S, Isaacs A, van Duijn CM: GenABEL: an R package for genome-wide association analysis. Bioinformatics 2007, 23:1294-6.

10. [http://qtl-mas-2012.kassiopeagroup.com/en/dataset.php].

doi:10.1186/1753-6561-8-S5-S4

Cite this article as: Minozzi et al.: Genome Wide Association Analysis of the 16th QTL- MAS Workshopdataset using the Random Forest machine learning approach. BMC Proceedings 2014 8(Suppl 5):S4.

\section{Submit your next manuscript to BioMed Central} and take full advantage of:

- Convenient online submission

- Thorough peer review

- No space constraints or color figure charges

- Immediate publication on acceptance

- Inclusion in PubMed, CAS, Scopus and Google Scholar

- Research which is freely available for redistribution

Submit your manuscript at www.biomedcentral.com/submit
Ciomed Central 